\title{
Simulación numérica por el método de elementos finitos del comportamiento de una mandíbula ante un dispositivo de tracción cervical mandibular
}

\author{
Juan Felipe Isaza Saldarriaga, Santiago Correa Vélez*, Samuel Roldán Restrepo
}

\begin{abstract}
Resumen Se describe la simulación numérica mediante el método de elementos finitos de una mandíbula ante las cargas ejercidas por una terapia de Tracción Cervical Mandibular (TCM). La geometría del problema se obtuvo a partir del procesamiento de imágenes de Tomografía de un niño de 12 años utilizando Rapid Form $2006^{\circledR}$. Se modelaron la mandíbula, 14 piezas dentales y los músculos masetero, temporal, pterigoideo interno y externo. Se aplicó una fuerza de 2,45 N sobre los primeros molares y las propiedades mecánicas usadas en el modelo fueron tomadas de la literatura. Se aplicaron restricciones en seis grados de libertad sobre el hueso temporal. El mallado del modelo fue realizado en GID 8.0.9 ${ }^{\circledR}$ y exportado para su análisis en ANSYS $10^{\circledR}$. Se obtuvo un modelo con 239.170 elementos y 356.237 nodos, se analizaron las tensiones y desplazamientos en diferentes planos con el propósito de compararlos con algunas tendencias de crecimiento observadas a nivel clínico sobre pacientes tratados con dicho dispositivo. Se encontraron varias concordancias entre la simulación y los resultados clínicos como el movimiento mandibular hacia abajo y hacia atrás. Además, se encontraron nuevas tendencias de desplazamientos del cuerpo mandibular que podrían ayudar a comprender resultados clínicos obtenidos a largo plazo que hasta la fecha no han tenido explicación.
\end{abstract}

Palabras-clave Bioingeniería, Elementos finitos, Ortodoncia, Tracción cervical mandibular.

\section{Finite element simulation of the jaw behavior under the action of a mandibular cervical headgear device}

\footnotetext{
Abstract This paper describes the numerical simulation, using the Finite Element Method, of a human jaw to evaluate the loads exerted by a Mandibular Cervical Headgear (MCH) therapy. The geometry of the problem was obtained from CT imaging of a twelve year old child using Rapid Form 2006 ${ }^{\circledR}$. The jaw, fourteen dental pieces, the masseter muscle, the temporal muscle and the internal and external pterygoid muscles were modeled. A load of $2.45 \mathrm{~N}$ was applied in the first molars and the mechanical properties used in the model were taken from literature. Constraints in six degrees of freedom were applied to the temporal bone. The mesh was defined in GID 8.0.9 ${ }^{\circledR}$ and exported for its analysis into ANSYS $10^{\circledR}$. A model with 239,170 elements and 356,237 nodes was obtained. Principal stresses and displacements in different planes were analyzed with the purpose of comparing them with some growth trends observed at clinical level. Correlations between numerical simulation and clinical results were found, such as downward and backward movement of the mandibular body. Moreover, there were new trends in the mandible movements that could help to understand clinical outcomes in the long term.
}

Keywords Bioengineering, Finite elements, Orthodontics, Mandibular cervical headgear. 


\section{Extended Abstract}

\section{Introduction}

The Chin Cup, Facemask and MCH are devices commonly used for the purpose of treating skeletal Class III malocclusion by means of applying loads to inhibit mandibular growth over time. From the above, the MCH is the least studied but is demonstrated through clinical trials to be effective against moderate mandibular growth. (Arun and Erverdi, 1994, 1997; Baccetti et al., 2007; Battagel and Orton, 1995; Gianelly, 1971; Joho, 1973; Orton et al., 1983; Rey et al., 2008). The question this work sought to answer was: Is it possible with the approach of a finite element model in the linear elastic range to know the tendencies of human jaw movement to the action of a $\mathrm{MCH}$ device? This question aims to discover how the patterns of movements alter the growth and development of craniofacial structures under the action of MCH. This knowledge could prevent maxillofacial surgery in a large number of patients and at the same time reduce the psychological effects arising from the facial deformity and malpositioned teeth (Ossa, 1997).

\section{Materials and Methods}

A study consisting of 867 head and neck transverse images of computed tomography (CT) was done on the selected patient. The cuts thickness was $0.5 \mathrm{~mm}$. The processing of images for subsequent $3 D$ reconstruction was carried out in RapidForm ${ }^{\circledR}$ (Figure 4). Parametric surfaces were created and exported to GID 8.0.9 ${ }^{\circledR}$, where some corrections were made in patches of surfaces, including sharp angles, fragmented and overlapping lines. The volumes that make up each craniofacial structure of interest, the assignment of mechanical properties under the jaw areas and geometric modeling of the muscles of mastication were also generated in GID 8.0.9 $9^{\circledR}$.

Volumes were created for the body of the mandible, temporal bone, the articular disc and fourteen teeth (Figure 5). Each volume was meshed in GID 8.0.9 ${ }^{\circledR}$ with high order tetrahedral elements (10 nodes). Lines representing the action of masseter, temporal, external and internal pterygoids muscles were modeled using cable elements. The periodontal ligament of the first molars was meshed with shell type elements of $0.25 \mathrm{~mm}$ thickness.
(Figure 8). The mechanical properties for the mandibular body were taken from the literature.

For muscles, boundary conditions were imposed restricting all degrees of freedom at the ends of each element to simulate the muscle fibers. The forces and moments exerted by the device were assigned in the model. They were placed on the nodes belonging to the outer facial surface of the first molars in order to represent the assembly of the $\mathrm{MCH}$ device to the band.

\section{Results}

It can be observed from a sagittal plane that the chin (Pogonion) shifted downward and backward. The Gonion shifted upward and backward. The Condylion shifted upward and forward (Figure 11).

From a frontal plane (Figure 12), it can be observed that coronoids tend to join, Gonions (Go) tend to separate and external poles of the condyle tend to aggregate. From an occlusal plane in Figure 13, it can be seen that the mandibular arch gets closed. The reduction of the stresses between the first molar and the alveolar bone, due to periodontal ligament was $16.5 \%$. The reduction of stresses between the condyle and the glenoid fossa, caused by the articular disc was $24.2 \%$.

\section{Discussion}

From the athors knowledge there are no studies in the literature that simulate the behavior of the human jaw with a procedure of MCH. Therefore, this work constitutes the first evidence on the use of FEM to study the effects of this device on humans. The numerical model developed, compared with the models available in the literature (Ausiello et al., 2002; Beek et al., 2000; Kimura et al., 2006; Magne et al., 1999; Nagasao et al., 2003; Ulusoy and Darendeliler, 2008), was of high quality in terms of anatomical representation of the different craniofacial structures and rigorous in terms of allocation of the mechanical properties. For the simulation of muscular restrictions, even taking into account the simplifications, this model is closer to real operating conditions of the craniofacial complex as compared to most studies which do not take them into account or assume them to be infinitely rigid (Basciftci et al., 2008; Holberg et al., 2007; Isaza and Correa, 2008; Kimura et al., 2006; Nagasao et al., 2003). 


\section{Introducción}

En el campo de la investigación en bioingeniería existe la limitación de realizar experimentos directamente sobre sujetos vivos ya que en algunos casos podría resultar costoso y éticamente cuestionable. Por tanto, es cada vez más importante la utilización de modelos numéricos que se aproximen a problemas reales, los cuales, dependiendo de la calidad de la simulación, pueden ser aplicados a la clínica.

Dentro de los métodos numéricos aplicables en bioingeniería existe el análisis por elementos finitos (Brekelmans et al., 1972), el cual permite estimar la respuesta de un medio continuo ante acciones externas, mediante la subdivisión del mismo en unidades elementales en las cuales se plantean en forma débil las ecuaciones diferenciales que gobiernan el problema físico. Una vez planteadas las ecuaciones diferenciales a nivel elemental, se ensamblan a nivel global, cumpliendo con los requisitos de continuidad (Taylor et al., 1986). Por último, el sistema matricial de ecuaciones resultante se resuelve, teniendo en cuenta las condiciones de contorno y las cargas externas (Bathe, 1982).

El prognatismo mandibular, un tipo de maloclusión conocida como Clase III, es causada por una combinación de factores intrínsecos como los genéticos y extrínsecos como el medio ambiente, la nutrición y el estado socioeconómico entre otros. Se caracteriza porque el arco dentario mandibular (inferior) se encuentra más adelantado que el arco dentario maxilar (superior), comprometiendo la estética facial y la adecuada función del sistema masticatorio (Figura 1). En algunos pacientes, este tipo de maloclusión se manifiesta a edad temprana y se hace necesario implementar diferentes tipos de tratamientos para la corrección ortopédica del desbalance en el crecimiento de los maxilares.

Los dispositivos comúnmente utilizados con el propósito de tratar la maloclusión Clase III esquelética, son una combinación de elementos removibles y fijos, los cuales permiten aplicar las fuerzas al complejo craneofacial para obtener la modificación del crecimiento deseada. Entre ellos se encuentran la mentonera, la máscara facial y la tracción cervical mandibular (TCM), siendo esta última una de las menos estudiadas, pero que ha demostrado ser efectiva en el tratamiento de este tipo de maloclusión cuando es originada por un crecimiento mandibular moderado (Arun y Erverdi, 1994, 1997; Baccetti et al., 2007; Battagel y Orton, 1995; Gianelly, 1971; Joho, 1973; Orton et al., 1983; Rey et al., 2008).

El dispositivo de TCM es un artefacto removible fabricado en acero inoxidable, en un perfil circular de diámetro 1,88 mm (Figura 2). Dicho dispositivo posee tres zonas principales, la primera (Punto A) es la zona de anclaje a los primeros molares del paciente mediante una banda metálica que los abraza. La segunda (Punto B) es la zona en donde ensambla un resorte que tiene como función ejercer una fuerza de 2,45 N (250 gr) (Mora et al., 2007) cuyo punto de apoyo se ubica en la parte posterior del cuello del paciente y que se nombra como (Punto C) (Figura 3).
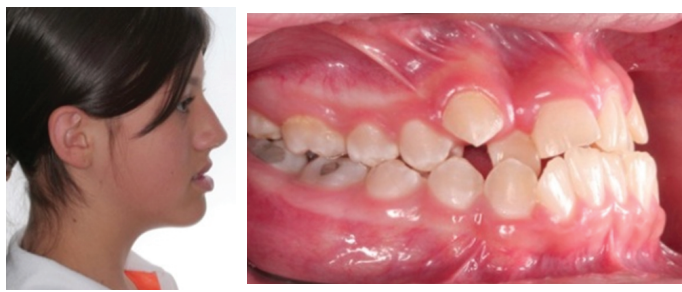

Figura 1. Paciente con maloclusión Clase III.

Figure 1. Class III malocclusion patient.

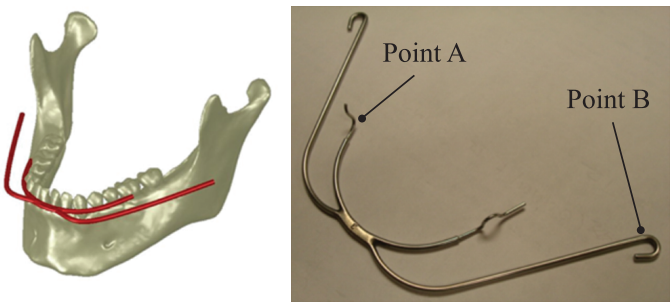

Figura 2. Dispositivo de TCM.

Figure 2. Mandibular Cervical Headgear (MCH) device.
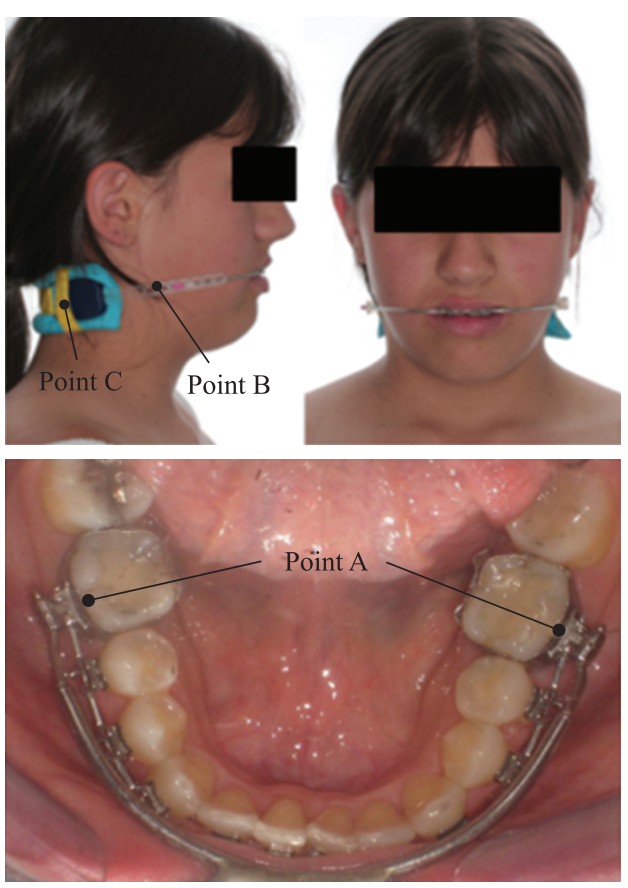

Figura 3. Paciente con dispositivo de TCM.

Figure 3. Patient with Mandibular Cervical Headgear (MCH) device. 
La pregunta que se buscó responder con este trabajo fue: ¿Es posible mediante el planteamiento de un modelo por elementos finitos en el rango elástico lineal conocer las tendencias de movimiento de una mandíbula humana ante la acción de un dispositivo de TCM? Esta pregunta tiene el propósito de descubrir como las tendencias de los desplazamientos alterarían el crecimiento y desarrollo de las estructuras craneofaciales bajo la acción de la TCM. El entendimiento de dichos efectos a largo plazo posibilitaría en un número considerable de pacientes el evitar que tengan que ser intervenidos con cirugía maxilofacial, procedimiento quirúrgico invasivo y costoso. Además, la posibilidad de evitar que los pacientes padezcan problemas sociales y psicológicos asociados a una sonrisa con dientes mal posicionados y la deformidad de su cara (Ossa, 1997).

Para resolver esta pregunta se planteó un procedimiento que, a partir del procesamiento de imágenes biomédicas en formato DICOM (Digital Imaging and Communications in Medicine), facilite y mejore la calidad geométrica de la anatomía mandibular y la correcta asignación de las propiedades mecánicas, cargas y restricciones, proporcionando una malla de elementos finitos que finalmente pueda ser utilizada en un software comercial.

\section{Materiales y Métodos}

\section{Reconstrucción 3D}

Se realizó un estudio de cuello y cabeza constituido por 867 Imágenes transversales de tomografía axial computarizada (TAC) sobre el paciente seleccionado. La distancia entre cortes fue de $0,5 \mathrm{~mm}$. El procesamiento de las imágenes para su posterior reconstrucción 3D fue llevado a cabo en el software RapidForm $2006^{\circledR}$ (Figura 4).

A partir de las superficies triangularizadas se crearon superficies paramétricas las cuales fueron exportadas a GID 8.0.9 ${ }^{\circledR}$ en donde se realizaron algunas correcciones en los parches de superficies, tales como ángulos agudos, líneas fragmentadas y superposición de puntos que en una eventual malla de elementos finitos causan concentración de elementos y discontinuidad nodal que imposibilita obtener mallas estructuradas, además de producir matrices globales singulares que hagan irresoluble el problema. En GID 8.0.9 $9^{\circledR}$ también se realizó la generación de los volúmenes que conforman cada estructura craneofacial de interés, la asignación de propiedades mecánicas según las zonas mandibulares y la modelación geométrica de los músculos de la masticación. Se crearon volúmenes para el cuerpo mandibular, el hueso temporal, el disco articular y catorce piezas dentales (Figura 5).

La banda por medio de la cual se ancla el dispositivo de TCM a los primeros molares se representó como una superficie, al igual que el ligamento periodontal (Figura 6). Los músculos de la masticación: temporal, masetero, pterigoideo interno y externo fueron modelados como un conjunto de líneas que simularon las fibras musculares. En la Figura 7 se observa la modelación del masetero y el temporal.
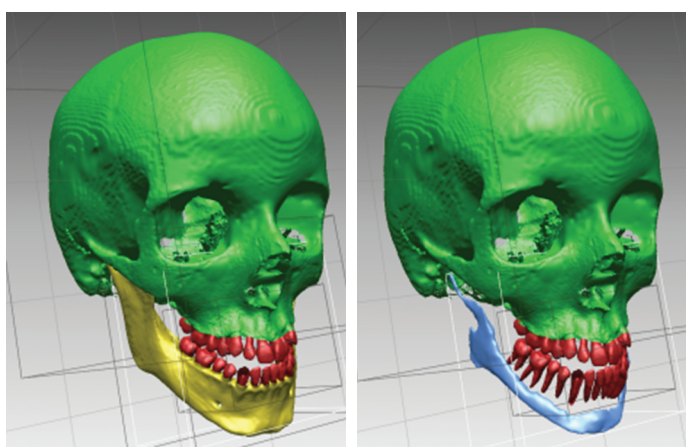

Figura 4. Reconstrucción 3D

Figure 4. 3D Reconstruction.
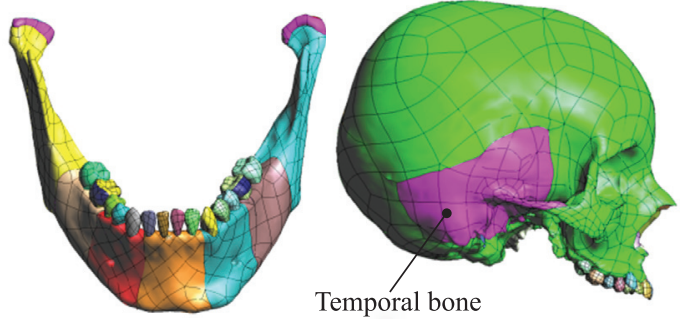

Figura 5. Generación de volúmenes.

Figure 5. Volume generation.

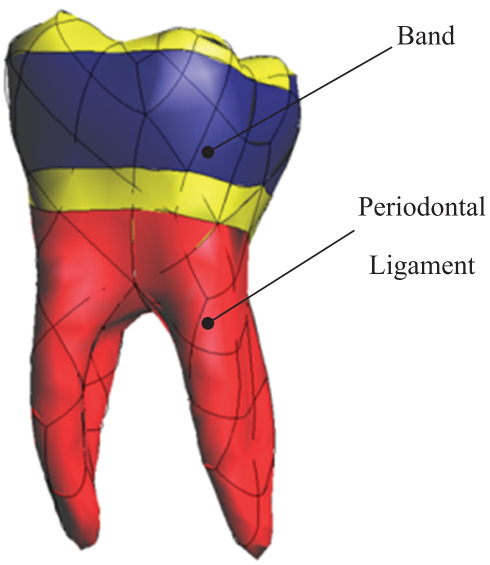

Figura 6. Banda y ligamento periodontal. Figure 6. Band and periodontal ligament. 
Una vez terminada la modelación geométrica del problema se procedió a generar el modelo de elementos finitos cuyo primer paso fue la generación de la malla en GID 8.0.9 ${ }^{\circledR}$.

\section{Modelo de elementos finitos}

\section{Mallado}

Cada volumen fue mallado en GID 8.0.9 ${ }^{\circledR}$ con elementos tetraedros de alto orden (10 nodos). Las líneas que representan la acción de los músculos masetero, temporal y pterigoideos externo e interno se mallaron como elementos cable (link 10) tridimensionales de 2 nodos. El ligamento periodontal de los primeros molares fue mallado con elementos tipo Shell de $0,25 \mathrm{~mm}$ de espesor, al igual que la banda de acero inoxidable con un espesor de 1,5 mm (Figura 8).

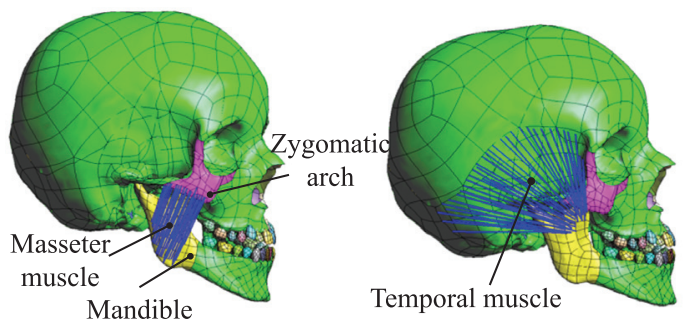

Figura 7. Músculos Masetero y Temporal. Figure 7. Masseter and temporal muscles.
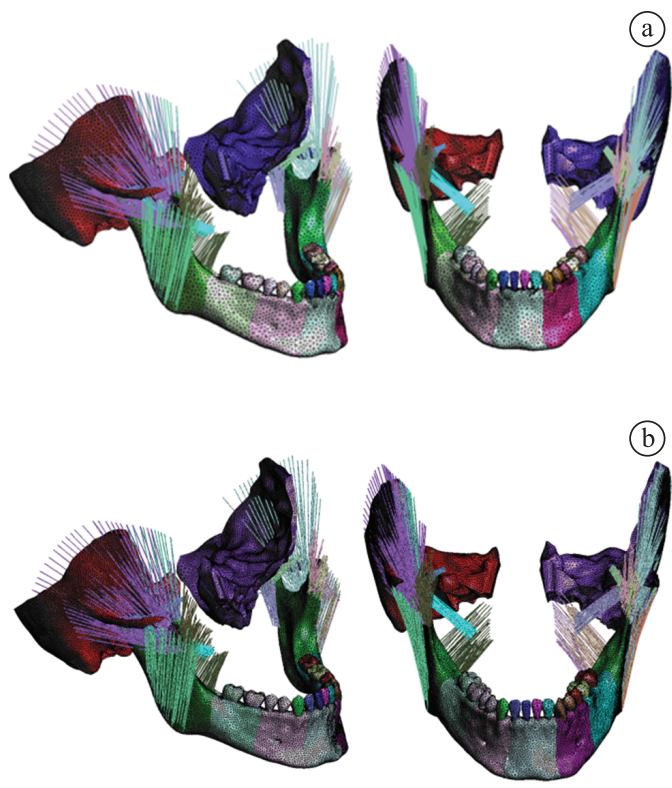

Figura 8. a) Malla de 239.170 elementos y 356.237 nodos; b) Malla de 441.235 elementos y 653.027 nodos.

Figure 8. a) Mesh of 239, 170 elements and 356,237 nodes; b) Mesh of 441,235 elements and 653,027 nodes.
Utilizando el lenguaje de programación de GID 8.0.9 ${ }^{\circledR}$ se generaron archivos de texto que contienen la información de la malla de elementos finitos, esto es, coordenadas nodales y conectividades elementales. Posteriormente estos archivos se leyeron con Matlab 6.0 ${ }^{\circledR}$ para generar un archivo de compatibilidad con ANSYS $10.0^{\circledR}$ en APDL ${ }^{\circledR}$ (Ansys Parametric Design Language). Este último archivo es leído directamente por ANSYS, obteniéndose la malla de elementos finitos en dicho programa, lista para imponer propiedades de material, condiciones de contorno y cargas. Con este procedimiento se realizaron dos mallas de elementos finitos para validar la convergencia de la solución. La primera malla posee 239.170 elementos y 356.237 nodos (Figura 8a). La segunda malla tiene 441.235 elementos y 653.027 nodos (Figura 8b).

\section{Propiedades mecánicas}

Las propiedades mecánicas para las diferentes estructuras del modelo fueron tomadas de la literatura y se presentan en la Tabla 1.

Según el estudio de Dechow et al. (1992) las propiedades mecánicas del cuerpo mandibular se dividen en cuatro zonas: rama, molar, premolar/ canino y sínfisis. Cada una de estas regiones posee propiedades mecánicas anisotrópicas. Además, la orientación de los ejes coordenados $(x, y, z)$ sobre los cuales se orientan las propiedades mecánicas son diferentes para cada zona mandibular. En cada porción de la mandíbula, el eje $x$ se orienta de manera tangente a la superficie mandibular. El eje $y$ es perpendicular al eje $x$ y apunta en dirección superior, paralelo a la sección transversal del hueso cortical. Finalmente el eje $z$ es perpendicular al plano $(x, y)$ cumpliendo la regla de la mano derecha. Las direcciones de las propiedades de material se introducen en el modelo asignando sistemas de coordenadas locales $(x, y, z)$ en cada porción del hueso mandibular y orientando dichos sistemas consistentemente. Así los sistemas de coordenadas elementales quedan orientados de acuerdo a las propiedades del material.

Debido a la dificultad de obtener datos confiables sobre las propiedades mecánicas de los músculos masticatorios que proporcionen una idea de su rigidez, se decidió utilizar el módulo de elasticidad del tendón tibial anterior, medido in vivo utilizando técnicas de ecografía en tiempo real (Maganaris y Paul, 1999). En cuanto al valor de la relación de Poisson $(\mu)$ para tejidos blandos del cuerpo humano, se reportan valores en la literatura que van desde 0,4 (Wu et al., 2003) hasta 0,5 (Srinivasan et al., 1992). Para este trabajo se tomó un valor intermedio de $\mu=0,45$, tal como se reporta en la Tabla 1. Para determinar el área de la 
Tabla 1. Propiedades mecánicas

Table 1. Mechanical properties.

\begin{tabular}{|c|c|c|c|c|c|c|c|c|c|}
\hline \multirow{2}{*}{ Material } & \multicolumn{3}{|c|}{ Módulo de Young (GPa) } & \multicolumn{3}{|c|}{ Relación de Poisson } & \multicolumn{3}{|c|}{ Módulo Cortante (GPa) } \\
\hline & $x$ & $y$ & $z$ & $x y$ & $y z$ & $x z$ & $x y$ & $y z$ & $x z$ \\
\hline \multicolumn{10}{|l|}{$\begin{array}{l}\text { Hueso cortical } \\
\text { (Dechow et al., 1992) }\end{array}$} \\
\hline Rama & 19,7 & 14 & 10,9 & 0,65 & 0,315 & 0,325 & 6,25 & 5,0 & 4,25 \\
\hline Molar & 19,5 & 13,6 & 10,25 & 0,34 & 0,29 & 0,21 & 6,25 & 5,9 & 4,15 \\
\hline Canino/premolar & 25,5 & 14,5 & 10,2 & 0,15 & 0,215 & 0,315 & 6,25 & 5,05 & 3,45 \\
\hline Sínfisis & 22,4 & 14,2 & 10,7 & 0,215 & 0,285 & 0,305 & 6,0 & 4,85 & 3,65 \\
\hline \multicolumn{10}{|l|}{$\begin{array}{l}\text { Hueso Esponjoso } \\
\text { (O’Mahony et al., 2000) }\end{array}$} \\
\hline Rama & 0,127 & 0,431 & 0,511 & 0,3 & 0,3 & 0,3 & 0,0488 & 0,0488 & 0,165 \\
\hline Molar & 0,988 & 0,047 & 0,091 & 0,3 & 0,3 & 0,3 & 0,38 & 0,38 & 0,18 \\
\hline Canino/premolar & 0,904 & 0,156 & 0,696 & 0,3 & 0,3 & 0,3 & 0,347 & 0,347 & 0,06 \\
\hline Sínfisis & 2,283 & 0,123 & 1,113 & 0,3 & 0,3 & 0,3 & 0,878 & 0,878 & 0,0473 \\
\hline $\begin{array}{l}\text { Hueso temporal } \\
\text { (Peterson y Dechow, 2003) }\end{array}$ & 13,4 & 14,0 & 23,4 & 0,52 & 0,19 & 0,2 & 4,7 & 5,3 & 7,1 \\
\hline $\begin{array}{l}\text { Disco articular } \\
\text { (Tanne et al., 1991) }\end{array}$ & 0,092 & 0,092 & 0,092 & 0,4 & 0,4 & 0,4 & 0,0328 & 0,0328 & 0,0328 \\
\hline $\begin{array}{l}\text { Ligamento Periodontal } \\
\text { (Provaditis, 2000) }\end{array}$ & 0,00068 & 0,00068 & 0,00068 & 0,49 & 0,49 & 0,49 & 0,23 & 0,23 & 0,23 \\
\hline $\begin{array}{l}\text { Dentina } \\
\text { (Kinney et al., 1999) }\end{array}$ & 16,3 & 16,3 & 16,3 & 0,25 & 0,25 & 0,25 & 6,2 & 6,2 & 6,2 \\
\hline $\begin{array}{l}\text { Tendón humano } \\
\text { (Maganaris y Paul, 1999) }\end{array}$ & 1,2 & 1,2 & 1,2 & 0,45 & 0,45 & 0,45 & 0,413 & 0,413 & 0,413 \\
\hline $\begin{array}{l}\text { Banda Dental } \\
\text { Acero Inoxidable 316L } \\
\text { (www.matweb.com) }\end{array}$ & 193 & 193 & 193 & 0,25 & 0,25 & 0,25 & 77,2 & 77,2 & 77,2 \\
\hline
\end{tabular}

sección transversal de cada fibra muscular, se usaron los valores del área de la sección transversal fisiológica (ASTF) de los músculos involucrados en el modelo (Tabla 2), los cuales fueron tomados del estudio de Van Eijden et al. (1997). El ASTF de cada músculo se dividió por el número de elementos tipo cable (link) que los representan. De esta forma la sumatoria de las áreas de los elementos que representan a cada músculo da como resultado el ASTF de cada uno sin importar el número de elementos que se hayan modelado. De esta forma la sumatoria de las rigideces de cada fibra muscular se corresponde con la rigidez del músculo completo.

Fuerzas y momentos ejercidos por el dispositivo de TCM

Según la práctica clínica, sobre el paciente se aplica una fuerza de 2,45 N (250 gr) (Mora et al., 2007) dirigida desde la región adyacente a las mejillas del paciente del dispositivo de TCM (Punto B) hasta la zona posterior del cuello (Punto C). Se determinaron las componentes tridimensionales de esta fuerza, mediante su vector director (Figura 9) y se trasladaron con sus momentos correspondientes a los nodos localizados en la superficie vestibular exterior de las bandas de acero ubicadas en los primeros molares, tal como se observa en la Figura 10.

\section{Restricciones}

El modelo de elementos finitos se restringió en todos los grados de libertad a nivel de la sutura escamosa del hueso temporal. Las fibras musculares fueron restringidas igualmente en todos los grados de libertad a nivel de la línea temporal inferior en el caso del músculo temporal, el arco cigomático en el músculo masetero, la gran ala del esfenoides y la placa pterigoide en el pterigoideo externo y finalmente el lado medial de la placa pterigoide y la apófisis piramidal del hueso palatino en el pterigoideo interno. Dichas restricciones pueden observarse en la Figura 10. La reacción de las fibras musculares ante la carga externa hace que el modelo sea auto-equilibrado.

\section{Resultados}

\section{Desplazamientos}

Las imágenes en las que se muestran resultados de desplazamientos tienen una escala de amplificación de 1.000 . 
Tabla 2. Área de la sección transversal de los músculos de la masticación.

Table 2. Cross sectional area of masticatory muscles.

\begin{tabular}{lccccc}
\hline \multicolumn{1}{c}{ Músculos } & ASTF & $\begin{array}{c}\mathbf{N}^{\mathbf{0}} \text { elementos } \\
\text { lado derecho }\end{array}$ & $\begin{array}{c}\mathbf{N}^{\mathbf{0}} \text { elementos } \\
\text { lado izquierdo }\end{array}$ & $\begin{array}{c}\text { Área por } \\
\text { elemento lado } \\
\text { derecho }\end{array}$ & $\begin{array}{c}\text { Área por } \\
\text { elemento lado } \\
\text { izquierdo }\end{array}$ \\
\hline Masetero & 6,82 & 44 & 47 & 0,155 & 0,145 \\
Temporal & 7,70 & 146 & 108 & 0,053 & 0,072 \\
Pterigoideo interno & 2,47 & 57 & 60 & 0,043 & 0,041 \\
Pterigoideo externo & 2,82 & 60 & 63 & 0,047 & 0,045 \\
\hline
\end{tabular}

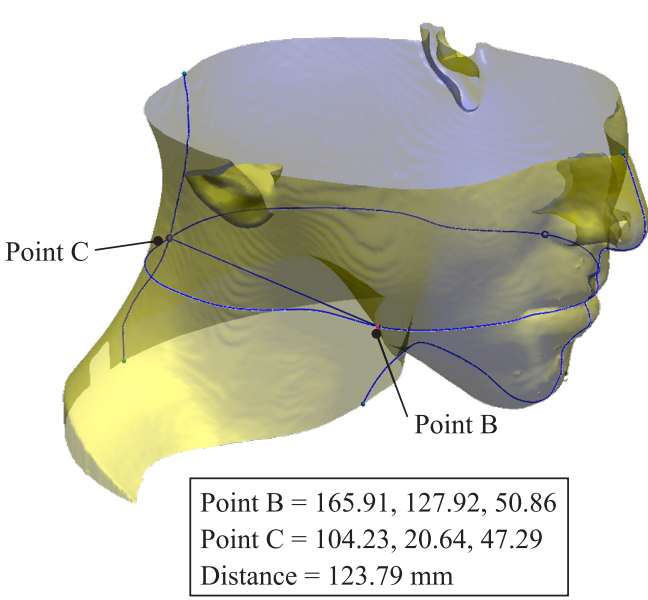

Figura 9. Vector director de la fuerza de TCM.

Figure 9. Force vector director in $\mathrm{MCH}$.

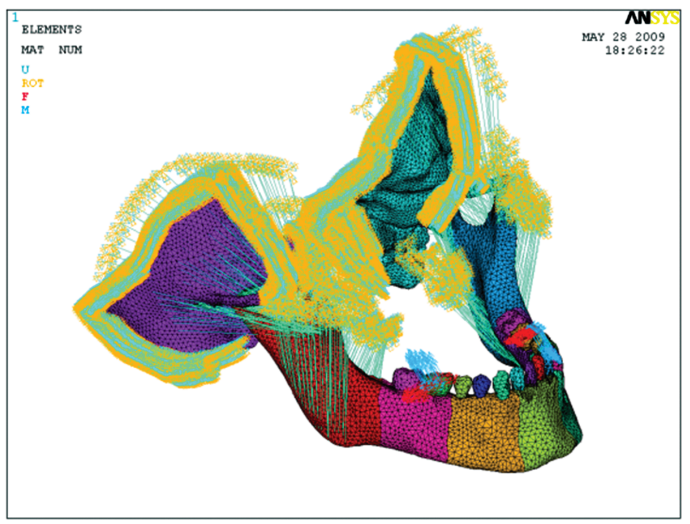

Figura 10. Fuerzas y restricciones del modelo.

Figure 10. Position vector of $M C H$ force.

\section{Plano sagital}

Se puede observar desde un plano sagital que la zona del mentón (Pogonion) se desplazó hacia abajo y hacia atrás. El Gonion se desplazó hacia arriba y hacia atrás. El Condylion se desplazó hacia arriba y hacia adelante (Figura 11).

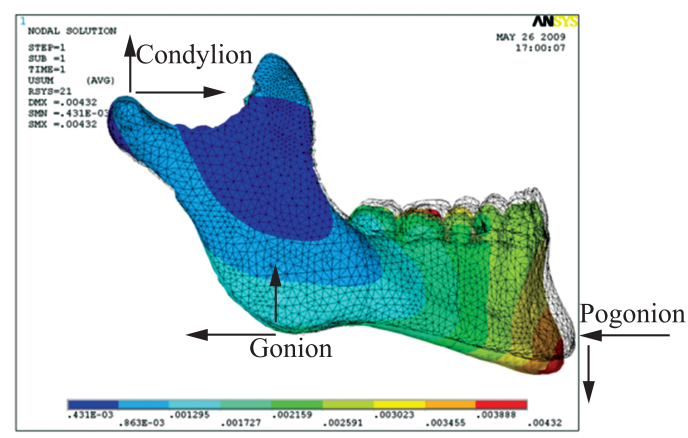

Figura 11. Desplazamientos desde un plano Sagital. Figure 11. Sagittal plane displacements.

\section{Plano frontal}

Desde un plano frontal en la Figura 12, se puede observar como las coronoides tratan de juntarse, el mentón baja, los Gonion (Go) izquierdo y derecho tratan de separarse y los polos externos del cóndilo tienden a juntarse.

\section{Plano oclusal}

Desde un plano oclusal en la Figura 13, puede observarse como el arco mandibular trata de cerrarse $y$ al igual que en el plano frontal se observa como las coronoides tratan de juntarse.

\section{Articulación temporomandibular (ATM)}

El desplazamiento que sufre el hueso temporal ante la carga del dispositivo de TCM a nivel de la fosa glenoidea (Figura 14) disminuye la carga efectiva sobre el disco articular, protegiendo su funcionalidad.

\section{Tensiones}

\section{Borde alveolar}

En la distribución de tensiones principales el máximo esfuerzo tensil (S1) fue de 2,44 MPa (Figura 15a), mientras que el máximo esfuerzo compresivo (S3) fue de 1,94 MPa (Figura 15b). La reducción de tensiones entre el primer molar y el hueso alveolar, debido al ligamento periodontal, fue de $16,5 \%$. 


\section{Disco articular}

En la región cóndilar del disco, la tensión compresiva (S3) fue de 0,124 MPa (Figura 16a). La tensión compresiva ( $\mathrm{S} 3$ ) en la región temporal del disco fue de 0,094 MPa (Figura 16b). La reducción de tensiones entre el cóndilo y la región temporal, debida al disco articular fue del $24,2 \%$.

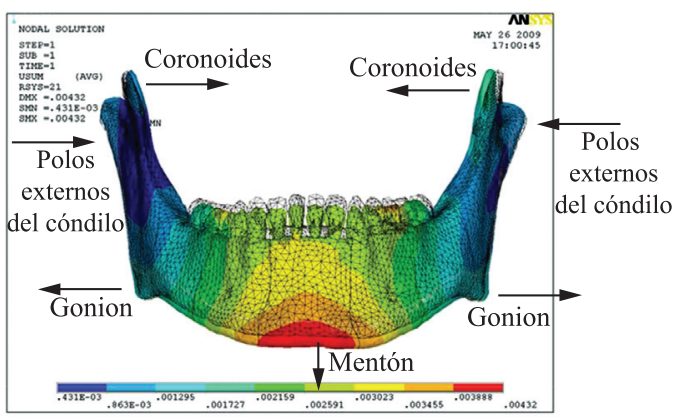

Figura 12. Desplazamientos desde un plano Frontal.

Figure 12. Frontal plane displacements.

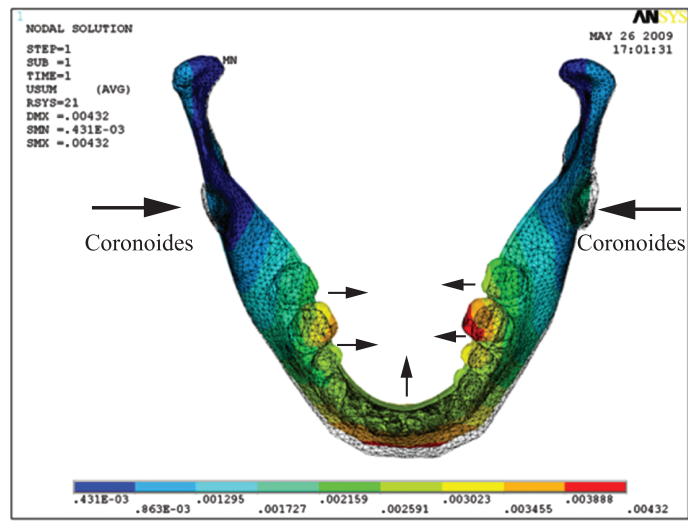

Figura 13. Desplazamientos desde un plano oclusal. Figure 13. Occlusal plane displacements.

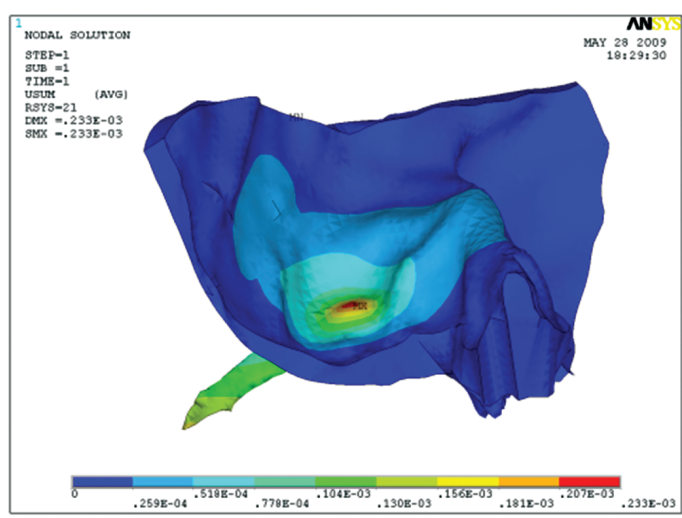

Figura 14. Articulación temporomandibular. Figure 14. Temporomandibular joint.

\section{Cuerpo y rama mandibular}

La máxima tensión tensil en el cuerpo y rama mandibular fue de $0,33 \mathrm{MPa}$ (Figura 17a), mientras que la máxima tensión compresiva fue de 0,6 MPa (Figura 17b). La presencia de tensiones compresivas en el aspecto vestibular y lingual del mentón, confirma la tendencia de estrechamiento del arco mandibular y la tendencia de los incisivos centrales a desplazarse en dirección anterior.

\section{Validación de la malla}

La Tabla 3 muestra los resultados de las tensiones principales (S1 y S3) en las tres regiones de análisis: borde alveolar, disco articular y cuerpo y rama mandibular, para las dos mallas de elementos finitos analizadas (Figuras 8a,b). Se observa que las diferencias entre los valores de tensión no superan el $5 \%$, con lo cual se garantiza la convergencia de la solución numérica.

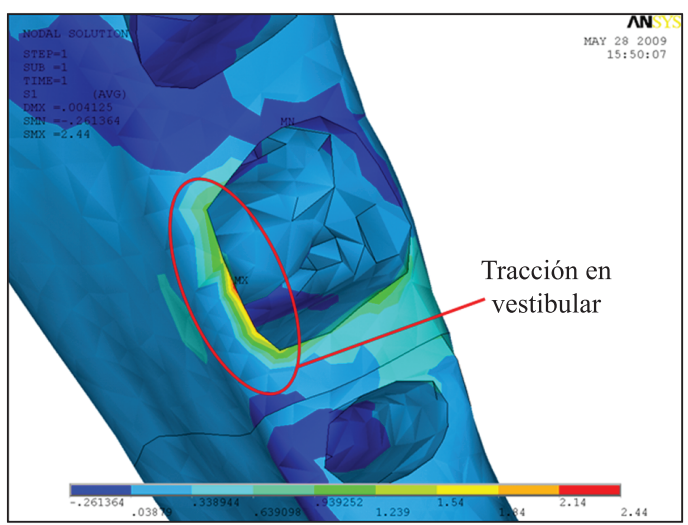

(a)

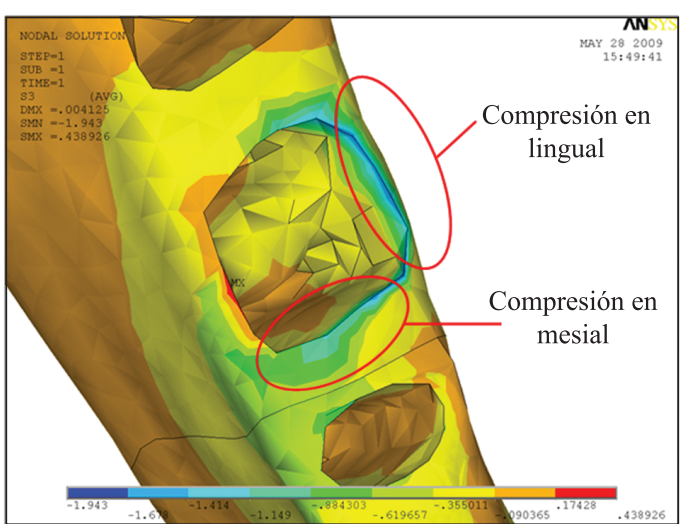

(b)

Figura 15. Tensiones principales en el borde alveolar: a) S1; b) S3. Figura 15. Principal Stress in the alveolar ridge: a) S1; b) S3. 


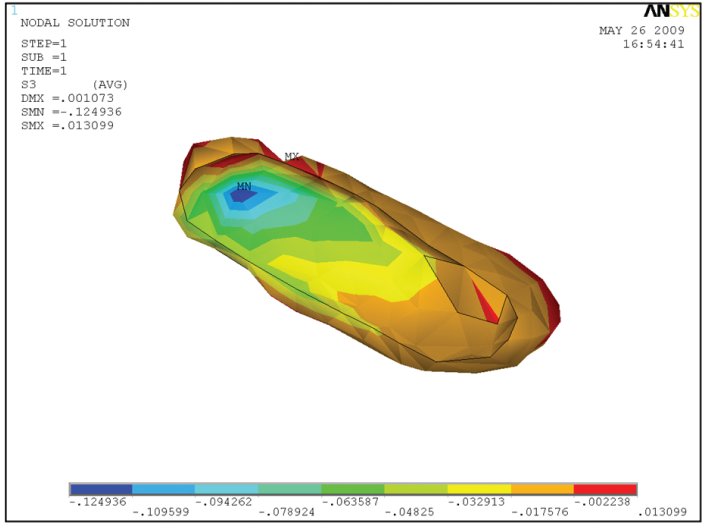

(a)

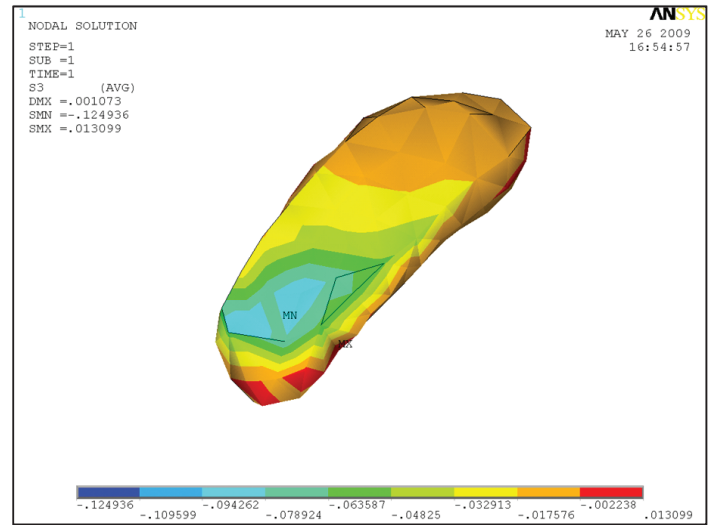

(b)

Figura 16. Tensiones compresivas (S3) en el disco articular: a) Región condilar; b) Región temporal. Figura 16. Compressive stress (S3) in the articular disc: a) Condilar Region; b) Temporal region.



(a)

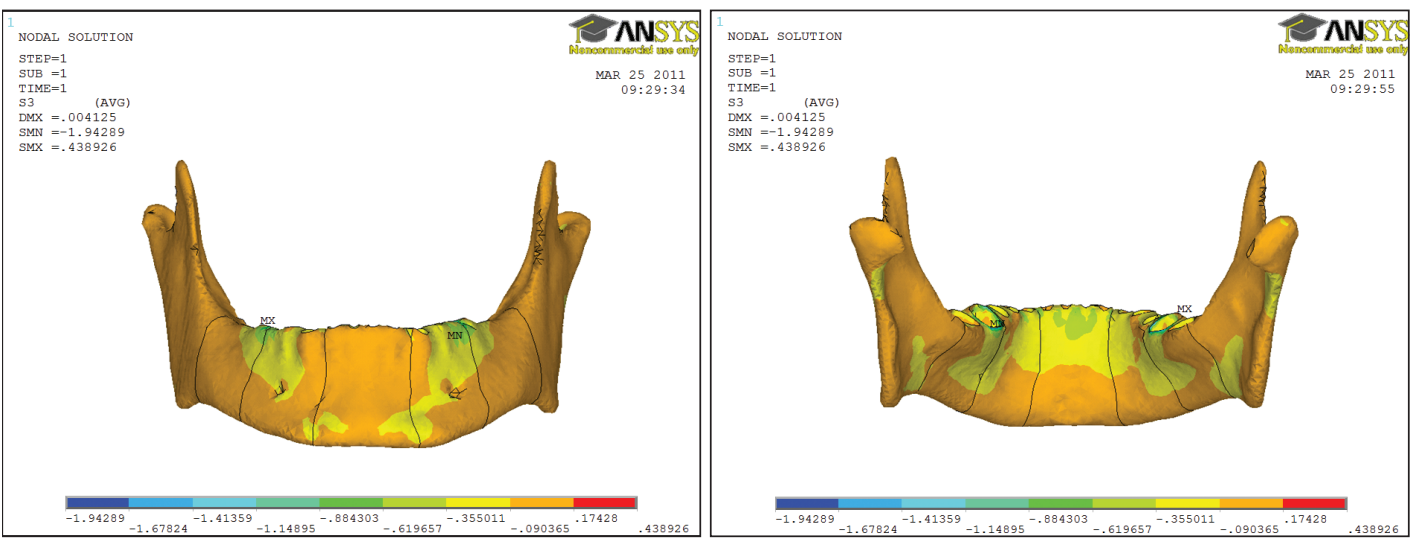

(b)

Figura 17. Tensiones principales en cuerpo y rama mandibular: a) $\mathrm{S} 1$; b) $\mathrm{S} 3$.

Figure 17. Principal stresses in body and mandibular ramus: a) S1; b) S3. 
Tabla 3. Validación de la malla de elementos finitos (NR: No Reportado).

Table 3. Finite element mesh validation.

\begin{tabular}{lccc}
\hline \multirow{2}{*}{ Región anatómica } & Malla a. & Malla b. & $\begin{array}{c}\text { Diferencia } \\
\mathbf{( \% )}\end{array}$ \\
\cline { 2 - 3 } & P1/P3 $\mathbf{M P a})$ & P1/P3 (MPa) & 4,5 \\
\hline Borde alveolar & $2,44-1,94$ & $2,55-2,02$ & 4,8 \\
Disco articular & $\mathrm{NR}-0,124$ & $\mathrm{NR}-0,130$ & 4,0 \\
Cuerpo y rama mandibular & $0,33-0,6$ & $0,34-0,62$ & \\
\hline
\end{tabular}

\section{Discusión}

En la literatura científica no existen estudios en donde se simule el comportamiento de la mandíbula humana ante un procedimiento de TCM, por lo tanto el presente trabajo se constituye en la primera evidencia sobre la utilización del FEM para el estudio de los efectos de este dispositivo sobre los seres humanos. El modelo numérico desarrollado, si se compara con los modelos disponibles en la literatura (Ausiello et al., 2002; Beek et al., 2000; Kimura et al., 2006; Magne et al., 1999; Nagasao et al., 2003; Ulusoy y Darendeliler, 2008) fue de gran calidad anatómica en cuanto a la representación de las diferentes estructuras craneofaciales y riguroso en cuanto a la asignación de las propiedades mecánicas. En cuanto a la simulación de las restricciones musculares, aun teniendo en cuenta las simplificaciones realizadas, este modelo se acerca en mayor medida a las condiciones reales de funcionamiento del complejo craneofacial si se compara con la mayoría de los estudios en donde no los tienen en cuenta o los asumen como infinitamente rígidos (Basciftci et al., 2008; Castaño et al., 2002; Holberg et al., 2007; Isaza y Correa et al., 2008; Kimura et al., 2006; Nagasao et al., 2003).

Los medios continuos fueron tratados en su totalidad como sólidos elástico lineales. Si bien no es la mejor aproximación, es lo que hasta el momento permite la omisión de las propiedades poroelásticas en el caso de los huesos y las propiedades viscoelásticas en el caso del disco articular. Es claro que futuros desarrollo deben ir orientados en este sentido para mejorar la respuesta del modelo.

\section{Conclusiones}

El modelo FEM confirmó algunas tendencias en cuanto a desplazamientos del cuerpo mandibular debidos al tratamiento de TCM. Se observó la rotación de la mandíbula alrededor del eje $z$ (mesiodistal) que produjo un desplazamiento del Gonion (Go) en dirección postero-superior y del Pogonion (Pog) en dirección postero-inferior. Estas tendencias han sido observadas a nivel clínico y se corresponden con lo observado en los estudios de Battagel y Orton (1995), y Orton et al. (1983). Sin embargo el estudio aporta tendencias de movimiento nuevas, no detectadas en radiografías cefalométricas bidimensionales. Como trabajo futuro resultaría interesante verificar estas tendencias de movimiento a nivel clínico.

El ligamento periodontal y el disco articular aportan una reducción de tensiones del 16,5\% y 24,2\% respectivamente a las estructuras óseas que los contienen. En el caso del disco articular los valores encontrados en este estudio son relevantes dado que podrían sustentar la hipótesis de que el tratamiento con TCM no altera la funcionalidad de la ATM, dado el porcentaje de reducción obtenido. Adicionalmente, el desplazamiento que sufre el hueso temporal ante la carga del dispositivo de TCM, disminuye la carga efectiva sobre el disco articular, protegiendo su funcionalidad. Este resultado concuerda con el estudio clínico realizado por Rey et al. (2008) en el cual no se encontraron síntomas de desordenes en la ATM en pacientes Clase III tratados con TCM.

Existe una falencia a nivel mundial sobre los valores de las propiedades mecánicas de algunas partes del cuerpo humano. Es importante avanzar en este aspecto para darle mayor validez a este tipo de estudios. Este trabajo se corresponde en gran medida con las tendencias de crecimiento observadas en la clínica, por lo tanto se podría afirmar que este tipo de simulaciones numéricas podrían ser utilizadas para el análisis previo de nuevos dispositivos de ortodoncia, que según los resultados podrían probarse clínicamente $\mathrm{y}$ traer como consecuencia un mejoramiento en los tratamientos de la maloclusión Clase III.

\section{Agradecimientos}

A COLCIENCIAS y al Grupo de Investigación en Bioingeniería GIB (EAFIT-CES).

\section{Referencias}

Arun T, Erverdi N. A cephalometric comparison of mandibular headgear and chin-cap appliances in orthodontic and orthopaedic view points. Journal of Marmara University Dental Faculty. 1994; 2(1):392-98. PMid:9582620. 
Arun T, Erverdi N. An alternative method to correct Class III malocclusion: early treatment. Turkish Journal of Orthodontics. 1997; 10(3):279-84.

Ausiello P, Apicella A, Davidson CL. Effect of adhesive layer properties on stress distribution in composite restorations a 3D finite element analysis. Dental Materials. 2002; 8(4):295303. PMid:11992906.

Baccetti T, Rey D, Angel D, Oberti G, Mcnamara JA. Mandibular cervical headgear vs rapid maxillary expander and face mask for orthopedic treatment of Class III malocclusion. Angle Orthodontist. 2007; 77(4):619-24. PMid:17605494.

Basciftci FA, Korkmaz HH, Uşümez S, Eraslan O. Biomechanical evaluation of chincup treatment with various force vectors. American Journal of Orthodontics and Dentofacial Orthopedics. 2008; 134(6):773-81. PMid:19061804.

Battagel JM, Orton HS. A comparative study of the effects of customized facemask therapy or headgear to the lower arch on the developing Class III face. European Journal of Orthodontics. 1995; 17(6):467-82. PMid:8682163.

Bathe JK. Finite Element Procedures in Engineering Analysis. New Jersey: Prentice Hall; 1982. p.225.

Beek M, Koolstra J, Van Ruijven LJ, Van Eijden TM. Three-dimensional finite element analysis of the human temporomandibular joint disc. Journal of Biomechanics. 2000; 33(3):307-16. PMid:10673114.

Brekelmans WA, Poort HW, Slooff TJ. A new method to analyze the mechanical behaviour of skeletal parts. Acta Orthopaedica Scandinavica. 1972; 43(5):301-17. PMid:4651051.

Castaño MC, Zapata U, Pedroza A, Jaramillo JD, Roldán S. Creation of a three-dimensional model of the mandible and the TMJ in vivo by means of the finite element method. International Journal of Computerized Dentistry. 2002; 5(2):87-99. PMid:12680039.

Dechow PC, Schwartz-Dabney CL, Ashman RB. Elastic properties of the human mandibular corpus. In: Goldstein SA, Carlson DS, editors. Bone biodynamics in orthodontic and orthopedic treatment. Ann Arbor, Michigan: University of Michigan Center of Human Growth and Development; 1992. p.2 99-314. Craniofacial Growth Series.

Gianelly A. Mandibular cervical traction in the treatment of Class I malocclusions. American Journal of Orthodontics. 1971; 60(3):257-63. PMid:5284932.

Holberg C, Mahaini L, Rudzki I. Analysis of sutural strain in maxillary protraction therapy. Angle Orthodontist. 2007; 77(4):586-94. PMid:17605502.

Isaza JF, Correa S. Metodología para la reconstrucción 3D de estructuras craneofaciales y su utilización en el método de elementos finitos. Ingeniería y Ciencia. 2008; 4(7):129-49.

Joho JP. The effects of extraoral low-pull traction to the mandibular dentition of Macaca mulatta. American Journal of Orthodontics. 1973; 64(6):555-77. PMid:4210181.
Kimura A, Nagasao T, Kaneko T, Tamaki T, Miyamoto J, Nakajima T. Adaquate fixation of plates for stability during mandibular reconstruction. Journal of Cranio-Maxillofacial Surgery. 2006; 34(4):193-200. PMid:16624567.

Kinney JH, Balooch M, Marshall GW, Marshall SJ. A micromechanics model of the elastic properties of human dentine. Archives of Oral Biology. 1999; 44(10):813-22. PMid:10530914.

Maganaris CN, Paul JP. In vivo human tendon mechanical properties. The Journal of Physiology. 1999; 521:307-13. PMid:10562354.

Magne P, Versluis A, Douglas WH. Rationalization of incisor shape: experimental-numerical analysis. The Journal of Prosthetic Dentistry. 1999; 81(3):345-55. PMid:10050124.

Mora DR, Oberti G, Ealo M, Baccetti T. Camouflage of moderate Class III malocclusions with extraction of lower second molars and mandibular cervical headgear. Progress in Orthodontics. 2007; 8(2):300-07. PMid:18030376.

Nagasao T, Kobayashi M. Tsuchiya Y, Kaneko T, Nakajima T. Finite element analysis of the stresses around fixtures in various reconstructed mandibular models - Part II (effect of horizontal load). Journal of Cranio-Maxillofacial Surgery. 2003; 31(3):168-75. PMid:12818603.

O’Mahony AM, Williams JL, Katz JO, Spencer P. Anisotropic elastic properties of cancellous bone from a human edentulous mandible. Clinical Oral Implants. 2000; 11(5):415-21. PMid:11168233.

Orton HS, Sullivan PG, Battagel JM, Orton S. The management of class III and class III tendency occlusions using headgear to the mandibular dentition. British Journal of Orthodontics. 1983; 10(1):2-12. PMid:6573194.

Ossa JA. La dentición como impacto social. Revista Facultad de Odontología Universidad de Antioquia. 1997; 8(2):24-28.

Peterson J, Dechow PC. Material properties of the human cranial vault and zygoma. Anatomical Record Part A. $2003 ; 274(1): 785-97$. PMid:12923889.

Provaditis CG. A comparative FEM-study of tooth mobility using isotropic and anisotropic models of the periodontal ligament. Medical Engineering \& Physics. 2000; 22(5):35970. PMid: 11121769

Rey D, Oberti G, Baccetti T. Evaluation of temporomandibular disorders in Class III patients treated with mandibular cervical headgear and fixed appliances. American Journal of Orthodontics and Dentofacial Orthopedics. 2008; 133(3):379-81. PMid:18331936.

Srinivasan MA, Gulati RJ, Dandekar K. In vivo compressibility of the human fingertip. Advances in Bioengineering. 1992; 22(1):573-76.

Tanne K, Tanaka E, Sakuda M. The elastic modulus of the temporomadibular joint disk from adult dogs. Journal of Dental Research. 1991; 70(12):1545-49. PMid:1774386.

Taylor RL, Simo JC, Zienkiewicz OC, Chan ACH. The patch test - a condition for assessing FEM convergence. International Journal for Numerical Methods in 
Engineering. 1986; 22(1):39-62. http://dx.doi.org/10.1002/ nme. 1620220105

Ulusoy C, Darendeliler N. Effects of Class II activator and Class II activator high-pull headgear combination on the mandible: a 3-dimensional finite element analysis study. American Journal of Orthodontics and Dentofacial Orthopedics. 2008; 133(4):490.e9-15. PMid:18405811.
Van Eijden TM, Korfage JA, Brugman P. Architecture of the human jaw-closing and jaw-opening muscles. The Anatomical Record. 1997; 248(3):464-74. PMid:9214565.

Wu JZ, Dong RG, Smutz WP, Schopper AW. Modeling of time-dependent force response of fingertip to dynamic loading. Journal of Biomechanics. 2003; 36(3):383-92. PMid:12594986.

\section{Autores}

Juan Felipe Isaza Saldarriaga, Santiago Correa Vélez

Grupo de Investigación en Bioingeniería, Departamento de Ingeniería de Diseño de Producto, Universidad EAFIT

Cra 49, No 7, Sur 50, Medellín, Colombia 